\title{
Hypervideo vs. Storytelling Integrating Narrative Intelligence into Hypervideo
}

\author{
Peter Hoffmann, Michael Herczeg \\ University of Luebeck \\ Institute for Multimedia and Interactive Systems \\ Ratzeburger Allee 160, 23538 Luebeck, Germany \\ \{hoffmann, herczeg\} a imis.uni-luebeck. de \\ http:// www. imis.uni-luebeck. de
}

\begin{abstract}
Hypervideo is one of several possible ways for interactive non-linear media. In its characteristics it is closely related to the purposes of digital narratives. The techniques of hypervideo could be used for the presentation of digitally told stories and vice versa. Many projects in both fields of work show the impressive possibilities each. But there seems to be a lack of using narrative intelligence in hypervideo. This paper shows how both fields of work could benefit from each other. Therefore two projects are introduced and their combination is discussed. The hypervideo environment HyPE includes an authoring tool, a stand-alone player for hypervideos and offers an API for the use in higher-ranking applications. Secondly the storytelling system called Jeherazade is introduced, which bases on the idea to enhance the classical theory of Aristotle to the new form of digital narrative. It is primarily developed for the use in presentations in distributed exhibitions but it is planned to be used for e-leraning as well.
\end{abstract}

Keywords: Hypervideo, non-linear media, interactive media, digital storytelling, narrative intelligence

\section{Introduction}

As hypervideo has two basic roots, the conventional video and hypermedia, there are several different definitions for it. One kind of definitions has its origins in hypermedia. Therefore it emphasises the aspect of augmenting information into digital video as for example Hesse does [9]. The user activates hyperlinks in the video and gets access to additional information to the actual scene in form of texts, images or even other videos. Another kind of definitions on the other hand emphasises the influence of the audience on the storyline [8]. This definition has its origins in storytelling. In this case the activation of a hyperlink by the viewer means that the viewer changes from one scene to another or from one perspective to another. So the audience does not follow the content in a linear but in a non-linear way; the audience creates an individual story-plot. None of both types of definitions is wrong, but in an ideal case hypervideo should include both: the usual interaction options like watching a video, fast-forward and fast-backward, stopping and the like, which derive from the medium "video", are combined by the possibilities of hypermedia 
and storytelling. So the surplus of hypervideo consists in the chance to break up the linearity of traditional video into a non-linear but still time-based medium. Furthermore the audience is no longer limited to the primary medium. The individual viewer is able to follow connections in the same video as well as connections to entirely different documents and media. So there is the chance of getting additional information to the basic video. Those connections are defined in form of hyperlinks as in any other hypermedia too.

As mentioned above, hypervideo offers the possibility to change from the linear order in a video to a non-linear order. The changing of the order is controlled by the viewer. The viewer has the opportunity to influence the course of the video on defined points-in-time. So the viewer can switch to another point-in-time than the actually presented point-in-time in the video or even to utterly different documents. Those documents can be other video-clips as well as other media-clips which are presented additionally to the actual video-clip.

Where hypervideo presents a movie with the embedded opportunity of the viewers interaction, digital storytelling goes on one step further. Braun [1] defines digital storytelling as "the telling of a story with an audience impact on the storyline, but without an impact on the story goal". This means that the audience has influence on the order in which the content is presented. All chosen and presented parts are conductive for reaching the goal, which is to be informed and/ or to acquire knowledge about a specific field in an entertaining and motivating manner.

The new form of "digital" or "virtual" storytelling follows the same well-known strategies similar to classical storytelling. The strategy most frequently used is the one which follows the course of suspense in a poetic story as Aristotle described it long ago. Following his ideas and theories, most narratives are divided in the four phases exposition, ascension, climax and conclusion [10, 11].

This more than 2000 years old concept is actually still in use and the basis for most plot in modern entertainment. One prominent example for the use of this theory gives Syd Field in his work [4]. Field follows strictly the ideas of Aristotle to include suspense to story plots even if gives the phases of the plot new names.

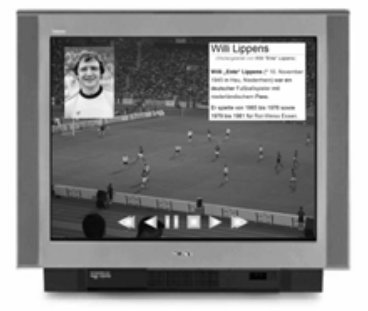

Fig. 1 - Augmented additional information and interaction options to change the order of points-in-time

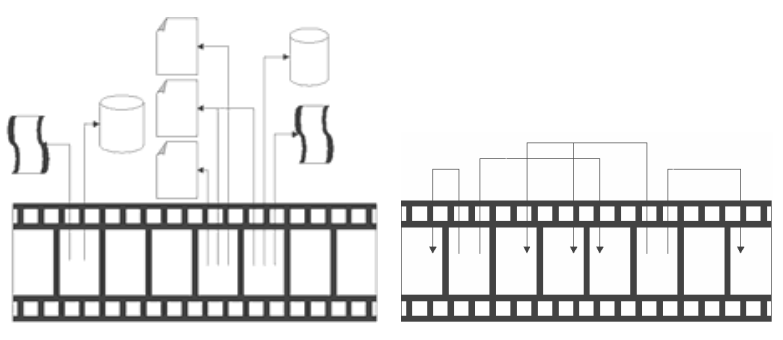

Fig. 2 - Different kinds of hypervideo definitions of: left: access to additional information right: access to other points-in-time or scenes

Digital storytelling offers another dimension which is not available in classical storytelling. A freedom of movement and a wider range of decisions is available in digital storytelling [9]. The new kind of narrative offers the choice between different 
narrative paths to the audience. The two dimensional story line does not longer exist in this method. It is expanded with at least one more dimension so that it can no longer be called a (story) line. So it is more precise to say, that the story told by using digital storytelling methods take place in a "story site" than to say it follows a single story line [14]. For supporting the viewer with a good guidance the storytelling system has to be intelligent in the narrative aspect [15]. The narrative intelligence decides which next parts of a story-site would fit best to the story experienced so far by the audience and gives the audience hints and clues how to get there.

Including a narrative intelligence into hypervideo should offer a big surplus to both, the viewer as well as to the author of hypervideos. The author is less limited in designing a content structure to the hypervideo. The viewer receives a presentation in form of a hypervideo which is adapted in its dramaturgy to the individual expectations.

\section{Problems in Design and Use of Hypervideo}

During designing and authoring hypervideo there are several different problems to be considered. The most important problem is the field of the interaction design. This includes both, placing additional information as well as providing information of the content structure.

With the option of augmenting additional media to the basic video the viewer of a hypervideo is able to reach more information as with a classical video. This is what hypervideo reaches for. But it is easily possible to overwhelm the visitor with too much augmented information or with information augmented at a badly chosen location on the screen. Furthermore the necessarily needed video links, the hotspots, appear and disappear during the time the video is presented. This might lead to a demotivation of the viewer. The viewer is possibly surprised by the appearing and disappearing signs which do not belong to the video itself. This detract the viewer from the context of the video and from experiencing it. It is mostly the task of the author of a hypervideo to avoid such problematic designs.

An even bigger problem is the design of the content structure and how to inform the audience about it. Usually in hypervideo it is possible for a viewer to jump between defined points-in-time in the basic video. With that option for example the opportunity is supported to view single scenes or even the whole video from different perspectives. An extensive problem comprises in the task to offer the information of the content structure to the viewer. So the viewer knos which point-in-time can be reached from the actual position or point-in-time. Actual two different ways to present this kind of information are commonly adopted.

The first one is to give a hint about the possible option to change to another scene or perspective. The viewer now has the opportunity to stop the video and to go back to a main menu. This shows the reachable scenes which can be jumped to. This is the way which most of the actual DVDs support. The big disadvantage of this kind of access to the content structure is that the immersion into the video is massively disturbed. The viewer has to stop the video for the interaction and therefore is distracted from the story line of the video. 
The second way of providing information commonly is to superpose a menu with all reachable scenes over the basic video. The video keeps running while the menu is shown as long as the interaction is possible. The story plot is not disturbed even the immersion into the video is affected by a possibly bothering menu. This is even more important when the menu is not really necessarily needed. In case the viewer does not want to switch to another scene or another perspective or does not want to get additional information, the menu is dispensable.
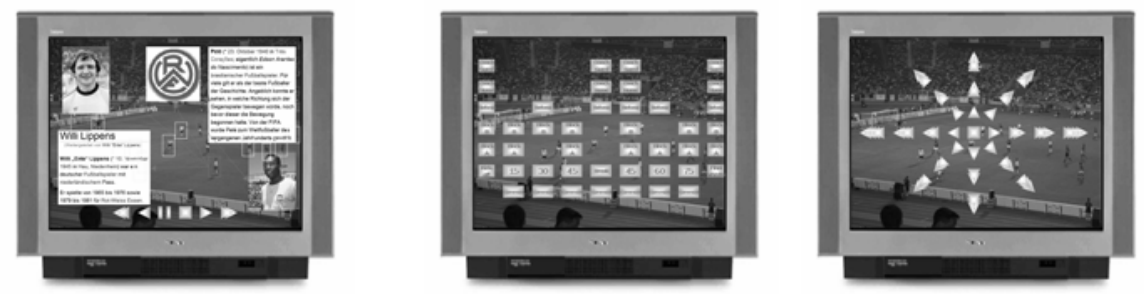

Fig. 3 - Problem of interaction design: placing additional information (left), information about content structure (middle), interaction with the content structure (right)

For considering the question if superposing a menu is necessary at a special pointin-time of the hypervideo it is furthermore essential that a hypervideo player has knowledge about the viewer. Only if the hypervideo player knows about the viewers target group, age, preferences, previous knowledge and most important about the viewers history in he hypervideo it can adapt the presentation individually. This means that not all defined hotspots make sense at any time of the presentation. So for example if a viewers' history leads to the conclusion, that the viewers most favoured topic is "architecture" it does not seem to be really reasonable to offer him a hotspot which leads to "philosophy". With fading in this hotspot the individual immersion of the viewers is again disturbed.

It would be a big advantage if hypervideo environments would support such a flexible way of access to additional media and to the content structure. The more adjusted the presentation is to the individual needs and preferences, the more entertaining and motivating is the hypervideo itself and its experience..

\section{Integrating Narrative Intelligence}

One way to reach a flexible access to hotspots respectively to the additional media and the content structure would be to connect a kind of ,narrative intelligence“ to a hypervideo environment. A problem to be solved for that way is, that most hypervideo players are not really open to be used by external applications. The hypervideo environment or at least the hypervideo player needs to understand messages the narrative intelligence sends to it and vice versa. 
At the Institute for Multimedia and Interactive Systems at the University of Luebeck two applications are in the deployment which fulfil the needs to reach an individual and a narrative supported hypervideo presentation.

\subsection{The Jeherazade Storytelling System}

The Jeherazade system is an interactive digital storytelling system which is based on the idea that audiences become story chasers $[11,12]$. Analog to the classical reader the audience in this new role of the chaser experiences a narrative by choosing an individual story path in a story site. The audience adopts an active role in the narrative: the audience chooses an individual story path and meets abstract and real characters who tell the information, give hints, and clues where to go and what to see next. The given information allows the audience to interact with the story line by making individual decisions.

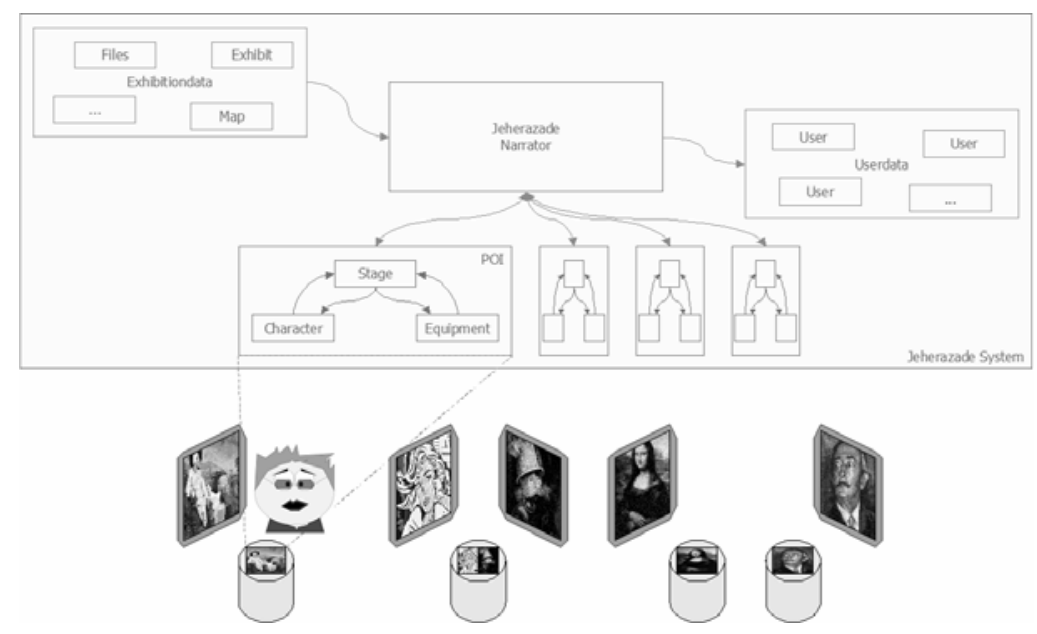

Fig. 4 - The Jeherazade storytelling Application

Characters in the Jeherazade system have the task to present the information stored in the story site. This is the way Jeherazade presents the story and gives hints and clues for the forthcoming story path. The Jeherazade system provides two kinds of characters. The first kind is called the story chaser. This character represents the audience and is therefore totally free to follow own individual paths through the story site. It does not present the stored information but informs the Jeherazade system about the audience's actions and choices. The other kind of characters in the Jeherazade system are called actors. Their special task is to present the narrative's content. Actors can appear in a wide range of different kinds. So an actor can be a personalized character like a $2 \mathrm{D}$ or a 3D avatar as well as an abstract character like a video or an audio player, a text or an image windows and so on [12].

The Jeherazade system uses equipment for the task of providing the interaction between the audience and the narrative. This is the way the audience "tells" the story 
site which story path he wants to choose or what kind of information he needs before he continues his story experience. As the characters too, the equipment in the Jeherazade system is also divided in two categories. The first kind of equipment builds the environment in which the story takes place, comparable to the scene or the stage in theatre or film. The other category of equipment supports the interaction especially the user's input. Analog to the characters the equipment also appears in a wide range of different kinds. So simple interaction devices like e.g. keyboard, mouse and joystick are supported as well as complex ones like speech input. As the Jeherazade system has a modular software architecture, it is easily possible to connect new and other kinds of characters and equipment to the system [12].

One possible application area of the Jeherazade system is the narrative support of presentations in distributed exhibitions. The visitor of a museum or the like can use the Jeherazade system as a guide through the exhibition. Therefore the system has several POIs where a Jeherazade stage is located. On the stages information to neighbouring exhibits are presented. The content and design of the local presentation depends on the visitors preferences, previous knowledge and the history in the exhibition. On the base of the information about the visitor and combined with the stored information about the exhibits the Jeherazade system calculates the best fitting exhibits for the further tour through the exhibition. The goal is to maintain a tour which follows an appealing and motivating dramaturgy in the order of the visited exhibits.

Other possible application areas are e-leraning and edutainment. Here a flexible and even more an individually adapted order leads to a higher motivation for zhe user to follow a lecture. The user is exactly postulated by his individual level that the best learning results can be reached. This is furthermore supported by the adaption of the presentation style to the users' target group.

\subsection{The HyPE Hypermovie Application}

The Hypervideo Environment HyPE is a hypervideo application, which supports both, the use as a stand-alone application as well as the simple integration into other projects. Therefore an environment with

- a stand-alone hypervideo player,

- an authoring tool

- $\quad$ and an open API to support the use of HyPE in external applications

was planned and realized. The first of those external applications which should use HyPE as an output device is Jeherazade. Because the Jeherazade system is implemented in Java it was obvious to start the realization of HyPE as well in Java, with all its supported interaction and media APIs for an easy connection of both.

A HyPE hypermovie consists of two parts, the video itself and the additional metadata file where the interaction possibilities are defined. The meta-data contains the annotation of the linkage to other time sequences and to other media. Therefore it was decided to be describe the meta-data in XML. This supports both, the readability and editability by (human) users as well as the automatically generation by appropriate editor applications. 


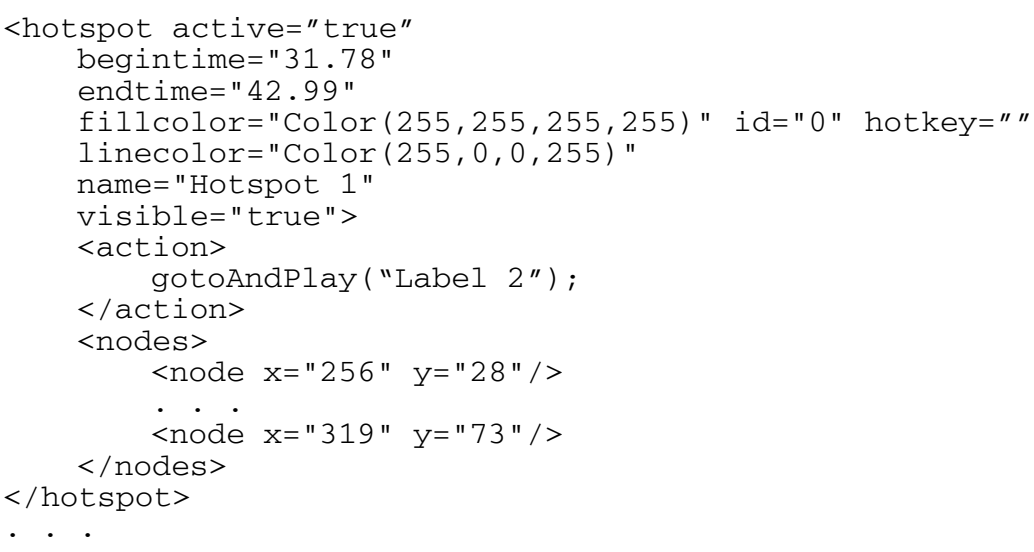

Fig. 5 - Source-Code example for the HyPE-API and the hotspot definition

The user's interaction in HyPE takes place via hotspots. An HyPE hotspot is an area the viewer can activate. This usually happens by a mouse click but can also take place by using keyboard shortcuts or the like. The hotspots can be defined in any way the author wants. So the author has influence

- on the geometry of the hotspot,

- on the visibility,

- on the time in the video, the hotspots appears and disappears,

- $\quad$ and on the actions which are activated with the hotspot.

Via the HyPE API it is possible to control all defined hotspots by an external application like the Jeherazade system. So it is possible for such external applications to set hotspots visible or active while the (hyper-) video is presented and to react on the activation of hotspots by the visitor individually.

\subsection{Hypervideo meets Storytelling: Connecting Jeherazade and HyPE}

Even hypervideo is more flexible than usual video though it is still more or less static:

- The author of a hypervideo defines an ambient structure for a basic video. In this structure the additional media are embedded by hotspots which link to this additional media.

- Furthermore the content structure is described by hotspots which link to points-in-time of the basic video which are related to the actual scene.

In general each time the hypervideo is started, the defined hotspots appear and disappear strictly at the defined time. This behaviour is independent of the viewers former choices. If for example the viewer followed in scene " $\mathrm{A}$ " a link to the pointof-time "B", this interaction is usually not stored. So it is not possible in the later presentation to react on this interaction. The history of the visitor, which could offer much information about his preferences, is lost.

By connecting an external "intelligence" like a storytelling application to the hypervideo environment the hypervideo gets a highly flexible structure in the content. 
After it is started the external storytelling application loads the meta-data information which describes the structure of the hypervideo and uses it in relation to the story parameters and audience parameters for the decisions of

- which hot spot has to be shown,

- which links to other points-in-time make sense for the actual audience and

- which additional media make sense for the actual audience .

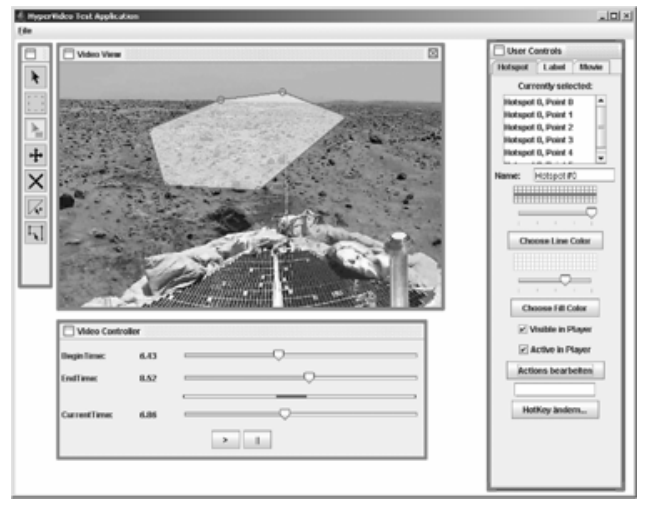

Fig. 6 - The HyPE-Application (Authoring tool including the hypervideo player)

The hypervideo player loads and starts the basic video and the meta data information. Even if the hypervideo player is driven by any external application the player needs to have the meta data information of the visdual parameters of the hotspots like their location on the screen, their collor and appearance. When reaching a point-in-time where the author had defined a hotspot, the player sends a message to the storytelling application. This decides whether the hotspot should be set visible and acitve or not and thus answers to the message. The knowledge about the hotspot and its target is described in the meta-data, the storytelling application loaded at the start.

Only if a hotspot is active an interaction by the viewer is possible. Is a hotspot activated by the viewer, the hypervideo player sends the message about the interaction also to the external storytelling application which starts the defined action for the activated hotspot.

Thus the hypervideo system (in this case: the hypervideo player plus the external narrative intelligence) has the opportunity to respond on the users behaviour. The presentation including the possible interactions is adapted to the history of the viewer. Furthermore the viewers preferences and previous knowledge, which is possibly known, can be considered for the actual presentation.

With the open API of the HyPE Hypervideo Environment the above mentioned idea of connecting external storytelling applications like the Jeherazade system is easily possible. The message objects send by the HyPE player can be received and processed in the Jeherazade system as well as vice versa the HyPE player can receive and process the message objects send by Jeherazade. 

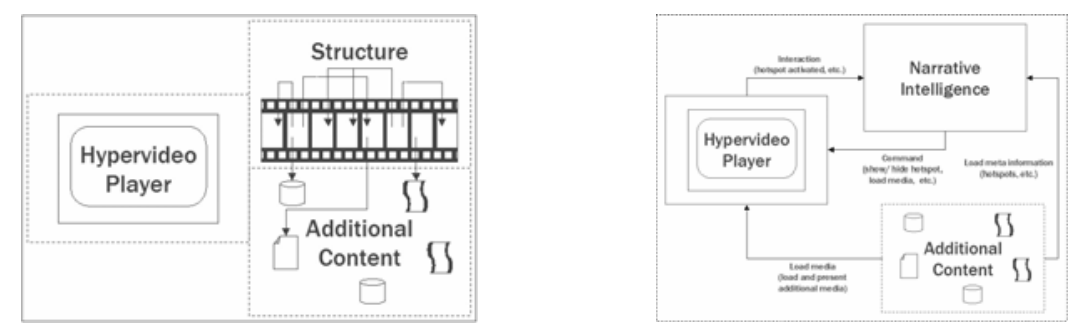

Fig. 7 - Hypervideo meets Storytelling

left: usual static linkage of content and scenes to a basic video right: an open API of the hypervideo player supports flexible linkage

\section{Conclusion and Future Prospects}

In this paper the problems of design and use of hypervideo was discussed. In particular the interaction with the content structure of a hypervideo was contemplated. To solve the resultant problems the possibility of connecting a narrative intelligence to a hypervideo environment was examined.

The deployment of the hypervideo environment HyPE was started for answering technical questions like the feasibility and capability of a Java based hypervideo application. In the further development of the HyPE environment aspects like the flexible and dynamic support of links and hotspots in the basic video was also considered. One special answer which resulted from the discussion about flexibility and dynamically shown hotspot was, that there should be an open API which can also be used by other applications.

With the Jeherazade storytelling system an application was available which fulfilled two aspects. On the one hand the Jeherazade systems offers a kind of narrative intelligence, on the other hand the Jeherazade system can use the HyPE player as a stage for itself. A presentation supported by Jeherazade follows in its order an dramaturgy which is individually adapted to the viewer. While both, Jeherazade and HyPE, support the usage of the same amount of different media and different media formats it was obvious to try to use the HyPE player as a possible stage for the Jeherazade system.

Therefore a demo hypervideo was produced. It introduces the viewer to the city of Luebeck, Germany and leads to several different places of interest. The viewer has the opportunity to choose his way in a totally free order. Furthermore the viewer can choose to receive additional information for each place.

The combination of the hypervideo environment and the external storytelling application in the demo movie offers good results. Hotspots are only shown when they are necessary and when they fit in the history of the viewer. So it is possible to support a dramaturgy into a hypervideo as originally mentioned. 

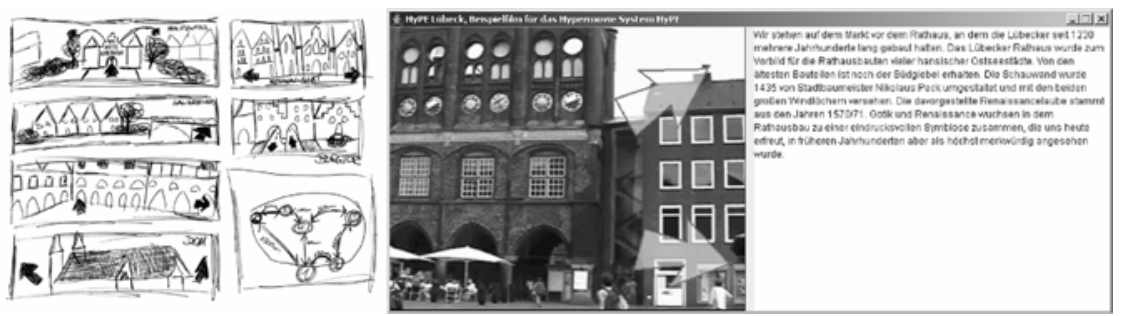

Fig. 8 - The HyPE stand alone player with a demo presentation of Luebeck

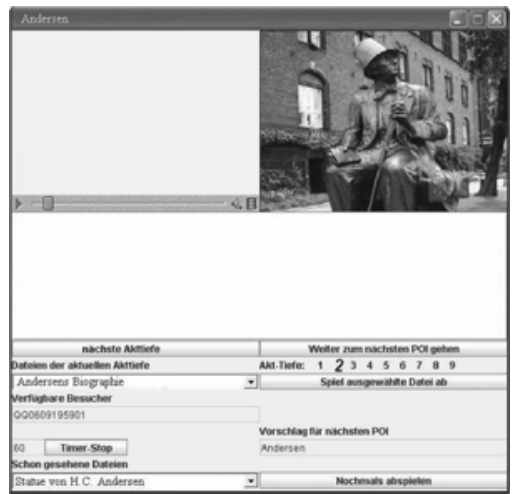

Fig. 9 - Prototypical stage of the Jeherazade system

\section{References}

1. Braun, N.. Kontrolliertes Erzählen von Geschichten mit integrierten, Videobasierenden Hyperstories. In: R. Keil-Slawik, H. Selke, G. Szwillus (Hrsg.): Mensch \& Computer 2004: Allgegenwärtige Interaktion. München: Oldenbourg Verlag. 2004, S. 157-167 (2004).

2. Braun, Norbert: Interaction Approach for Digital Video Based Storytelling. 9-th International Conference in Central Europe on Computer Graphics, Visualization and Computer Vision'2001, WSCG 2001, University of West Bohemia, Campus Bory, PlzenBory, Czech Republic, February 5-9, 2001

3. Doherty, J \& Girgensohn, A. \& Helfman J. \& Shipman F. \& Wilcox L. (2003). DetailonDemand Hypervideo. Palo Alto: FX Palo Alto Laboratory.

4. Field, Syd: Screenplay: The Foundation of Screenwriting (2005). Dell Publishing co. Inc. New York.

5. Finke, M. \& Wichert, R. (2003). Augmented Reality and Hypervideo Supporting Distributed Communities for Education \& Training. Darmstadt: Zentrum für Graphische Datenverarbeitung e.V.

6. Francisco-Revilla, L. (1998). A Picture of Hypervideo Today. Texas A\&M University. (www. csdl.tamu.edu/ 10f0954/ academic/ cpsc610/ p-1.htm 20.03.2006). 
7. Fransecky, R. \& Debes, J. (1972). Visual Literacy: A way to learn - A Way to Teach. Washington: Association for Educational Communications and Technology.

8. Girgensohn, A. \& Shipman, F. \& Wilcox, L. (2003). Hypervideo Summaries. Palo Alto: FX Palo Alto Laboratory

9. Heintze, J. v., Bode, C.: Emergent Stories - Eine neue Herangehensweise zum Erleben und zum Authoring interaktiver Geschichten. In: Spierling, Ulrike (Hrsg.): Digital Storytelling Tagungsband. Stuttgart : Fraunhofer IRB Verlag, 2000, S. 13-28 (Computer Graphik Edition 02)

10. Hesse, W. (2005). Didaktisches Design, Mediengestaltung, Visualisierung. (http://www.eteaching.org/didaktik/gestaltung/visualisierung/hypervideo, 20.03.2006).

11. Hoffmann, P., Herczeg, M.: Distributed Storytelling for Narrative in Spacious Areas. In Göbel, St., Braun, N., Spierling, U., Dechau J. \& Diener, H. (Eds.) Proceedings of Technologies for Interactive Digital Storytelling an Entertainment, Computer Graphik Edition, Band 9.Stuttgart: Fraunhofer IRB Verlag. pp. 346-350.

12. Hoffmann, P., Herczeg, M.: Expanding the storyline. In Bearman, D. \& Trant, J. (Eds.), Museums and the Web 2004, Proceedings. Toronto: Archives \& Museum Informatics.1. Baldonado, M., Chang, C.-C.K., Gravano, L., Paepcke, A.: The Stanford Digital Library Metadata Architecture. Int. J. Digit. Libr. 1 (1997) 108-121

13. Hoffmann, P.; Herczeg, M.: Distributed Storytelling for Narrative in Spacious Areas. In: TIDSE, 1st International Conference on Technologies for Interactive Digital Storytelling and Entertainment (Hrsg.). Darmstadt, 24. - 26. März 2003

14. Hoffmann, P.; Herczeg, M.: Expanding the storyline. In Bearman, D. \& Trant, J. (Eds.), Museums and the Web 2004, Proceedings. Toronto: Archives \& Museum Informatics (2004).

15. Mateas, Michael, Senger, Phoebe: Narrative Intelligence (Advances in Consciousness Research Series). American Association for Artificial Intelligence. Benjamins, John Publishing Company (1998).

16. Sawhney, N. \& Balcon, D. \& Smith, I (1996). HyperCafe: Narrative and Aesthtetic Properties of Hypervideo. Atlanta: Hypertext '96. (www. cs.unc.edu/ barman/ HT96/P24/ HyperCafe.html) [31.08.05] 